\title{
Impaired radial artery compliance in normotensive subjects with familial hypercholesterolemia
}

\author{
Cristina Giannattasio ${ }^{a}$, Arduino A. Mangoni ${ }^{\mathrm{b}}$, Monica Failla ${ }^{\mathrm{a}}$, Stefano Carugo ${ }^{\mathrm{b}}$, \\ Maria Luisa Stella ${ }^{b}$, Patrizia Stefanonic, Guido Grassib, Carlo Verganic, \\ Giuseppe Mancia ${ }^{\mathrm{a}, \mathrm{b}, \mathrm{d}, *}$

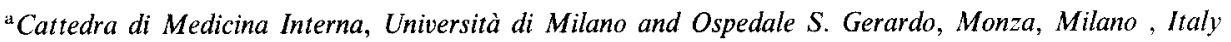

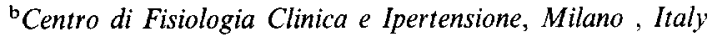 \\ Cattedra di Geriatria, Ospedale Maggiore and Università di Milano, Milano, Italy \\ ${ }^{\mathrm{d}}$ Centro Auxologico Italiano, Milano, Italy
}

Received 6 October 1995; revised 13 February 1996; accepted 23 February 1996

\begin{abstract}
Hypercholesterolemia impairs arteriolar dilatation, but whether the vascular abnormalities accompanying this condition include large artery function is unknown. We addressed this issue in 13 normotensive subjects with familial hypercholesterolemia (serum cholesterol $401.6 \pm 16.9 \mathrm{mg} / \mathrm{dl}$, mean \pm S.E., FHC) and no evidence of atherosclerotic lesions, in whom radial artery (RA) diameter and blood pressure (BP) were measured beat to beat by an echotracking and a Finapres device, respectively. RA compliance (RAC) was derived from the diameter/BP relationship and expressed over the systo-diastolic BP range, both at baseline and after a 12-min brachial artery occlusion. RAC was expressed also as the area under the RAC/BP curve divided for pulse BP. Measurements included maximal forearm blood flow (plethysmography) and minimal forearm vascular resistance (FVR) which were obtained from the values following the 12-min brachial arterial occlusion. Data were collected before and after 6- and 24-month lipid lowering treatment (simvastatin $40 \mathrm{mg} /$ day). Ten age-matched normotensive normocholesterolemic healthy subjects $(\mathrm{N})$ served as controls. Compared to N, baseline RAC was strikingly reduced in FHC $(-53.5 \%, P<0.01)$. After ischemia RAC increased significantly and markedly in $\mathrm{N}(+38.7, P<0.01)$, while only a modest and non-significant increase was observed in FHC. Minimal FVR was markedly higher in FHC than in N (3.5 \pm 0.9 vs $1.6 \pm 0.1$ units, $P<0.01)$. In FHC (7 subjects) RAC remained unchanged after 6 months of lipid lowering treatment, but increased markedly $(+55.2 \%$, $P<0.05$ ) when treatment was prolonged to 24 months. Lipid lowering treatment also reduced minimal FVR, the effect being significant both after 6 and after 24 months. No changes in RAC and minimal FVR were seen after 6 months in controls. Thus, in subjects with a marked increase in serum cholesterol due to FHC, not only arteriolar dilatation, but also RAC and distensibility are markedly impaired. This impairment can be favourably affected by an effective lipid lowering treatment of long duration.
\end{abstract}

Keywords: Familial hypercholesterolemia; Arterial compliance; Peripheral circulation; Lipid profile

\footnotetext{
* Corresponding author. Divisione Medicina Interna I, Ospedale S. Gerardo dei Tintori di Monza, Via Donizetti 106,20052 Monza, Milano, Italy. Tel.: + 3939 2333357; Fax: + 3939322274.
} 


\section{Introduction}

Several studies have shown that in normotensive subjects with a high serum cholesterol concentration the marked reduction in forearm vascular resistance (FVR) which follows prolonged ischemia or intra-arterial infusion of acetylcholine and/or metacholine is impaired [1 6]. This suggests that hypercholesterolemia alters the ability of the arterial vessels to dilate in response to metabolic or pharmacologic stimuli. It also suggests that this alteration originates from interference with the secretion and/or the vascular effects of endothelial relaxing factors.

No information exists as to whether the vascular abnormality characterizing normotensive hypercholesterolemic subjects also involves larger arteries, thereby affecting not only modulation of systemic vasomotor tone, but also arterial compliance. This is an important issue because a reduction in arterial compliance increases the traumatic effect of blood pressure (BP) on the vessel wall and enhances the rate of development of atherosclerosis $[7,8]$. In the present study we addressed this issue by measuring both forearm arteriolar dilatation after prolonged ischemia, compliance and distensibility of the radial artery (RA) in normotensive subjects with familial hypercholesterolemia (FHC). The study was performed before and after 6 and 24 months of administration of a hypocholesterolemic drug to further examine whether the vascular abnormalities are reversible by lipid lowering treatment.

\section{Methods}

\subsection{Subjects}

We investigated 23 outpatients with an age ranging from 45 to 53 years. Ten subjects ( 6 males and 4 females) were normocholesterolemic controls, while the remaining 13 subjects ( 6 males and 7 females) had a FHC of a heterozygote type as indicated by (1) a plasma low-density lipoprotein (LDL) cholesterol concentration greater than 200 $\mathrm{mg} / \mathrm{dl}$, (2) a plasma triglyceride concentration less than $110 \mathrm{mg} / \mathrm{dl}$ and (3) a serum cholesterol con- centration greater than $300 \mathrm{mg} / \mathrm{dl}$, (4) a tendon or an eye xantoma and (5) a history of premature coronary heart disease in at least one first-degree relative [9]. The ages of the control group and of its male and female components were $45.4 \pm 2.2$, $44.3 \pm 3.3$ and $47.0 \pm 3.3$ years, respectively. The corresponding figures for the FHC group were $47.1 \pm 2.5,46.8 \pm 3.7$ and $47.2 \pm 3.6$ years. All subjects had BP values less than 140/90 $\mathrm{mmHg}$ at repeated sphygmomanometric measurements. In the control group body mass index was $23.5 \pm 1.5 \mathrm{~kg} / \mathrm{m}^{2}$ and 4 subjects were smokers. The corresponding figures in the hypercholesterolemic group were $22.5 \pm 0.7$ and 4 . No female subject was in menopausal status or under hormonal treatment. No subject had a history of cardiovascular disease and physical or laboratory evidence of cardiovascular damage (baseline and exercise electrocardiogram, echocardiogram, Holter EKG, echo-Doppler of carotid and femoral arteries, etc.). FHC subjects were under dietary and drug (a statin, a fibrate or a resin) treatment. Drug treatment was withdrawn 2 months before the study. All subjects volunteered their participation in the study after being informed of its nature and purpose. The protocol of the study was approved by the Ethical Committee of the Institutions involved.

\subsection{Arterial compliance and distensibility}

Arterial compliance was measured in the left RA by an A-mode echo-tracking device (NIUS 01, Asulab, Neuchatel, Switzerland) [10]. The device consisted of a $10-\mathrm{MHz}$ focalized transducer which was stereotaxically positioned over the RA $2-4 \mathrm{~cm}$ above the wrist, direct contact with the skin (and arterial deformation) being prevented by use of a gel medium. With the subject supine and the left arm immobile at heart level, the transducer was switched to the Doppler mode and oriented perpendicularly to the longitudinal axis of the vessel. After switching to A-modc, the backscattered echoes from the inner anterior and posterior walls were visualized on an oscilloscope and the related radiofrequency signals were picked up by an electronic tracer whose displacement allowed evaluation of the vessel internal 
diameter 300 times per s. Continuous recording of RA diameter was coupled with continuous recording of BP by a photopletysmographic device (Finapres, Ohmeda, Englewood, CO) positioned on the middle finger of the ipsilateral hand and capable of providing BP values similar to the ones taken invasively from the RA and to accurately reproduce rapid and marked $\mathrm{BP}$ changes $[11,12]$. The arterial diameter and BP signals were directed to a computer which was programmed (1) to provide diameter-pressure curves over the range of $\mathrm{BP}$ values obtained by the finger recording, (2) to calculate cross-sectional compliance based on the arctangent model of Langewouters et al. [13], (3) to derive compliance-pressure curves also over the BP range obtained by the finger pressure recording, and (4) to also derive distensibility (compliance divided by diameter)pressure curve over the BP range obtained by the finger pressure. The echo-tracking device resolution allowed to identify diameter changes $\geq 150$ $\mu \mathrm{m}$, while the finger pressure device resolution allowed to identify BP changes $>2 \mathrm{mmHg}$. The within investigator variability of the RA diameter measurements, i.e. the coefficient variability of two measurements performed by the same operator in two different days, was $4 \%$. The RA diameter, RAC and BP measurements were associated with heart rate measurements which were obtained via the finger-pressure signal as the reciprocal of the interval between consecutive systolic peaks. All measurements were obtained in baseline condition and following prolonged occlusion of the brachial artery (see below), a procedure which increases RAC thus allowing to examine its response to a physiological stimulus [14-17].

\subsection{Blood flow and vascular resistance}

A venous occlusion pletysmograph (Hokanson Instruments, Issaquah, WA) was applied to the left forearm placed at the heart level, the mercury in sylastic strain-gauge being positioned $4-5 \mathrm{~cm}$ below the antecubital crease. Forearm blood flow was recorded on a polygraph (Grass Instruments, Quincy, MA) while circulation to the hand was excluded by means of a supra-systolic pressure in a cuff encircling the wrist. BP was measured by the above-mentioned finger device and FVR was calculated as the ratio between mean arterial pressure (diastolic BP + one third of pulse pressure) and forearm blood flow. All measurements were obtained in baseline condition and after the prolonged forearm ischemia caused by occlusion of the brachial artery as described by Folkow et al. [18] (see below). This was done to obtain maximal dilatation and thus a minimal resistance value.

\subsection{Protocol and data analysis}

The study was conducted in the afternoon following a 24-h abstinence from smoking and coffee consumption. The protocol of the study was as follows: (1) each subject was brought to the laboratory, placed in the supine position and fitted with the BP measuring and the echo-tracking device, (2) after a 20-min interval, BP, heart rate, diameter-pressure curves and compliance-pressure curves were measured continuously for $15 \mathrm{~min}$, (3) a suprasystolic pressure was applied for $12 \mathrm{~min}$ to the left arm to occlude the brachial artery and all hemodynamic measurements mentioned above were obtained again during the $5 \mathrm{~min}$ which followed the release of the occlusion [14-17]; the measurements were started $30 \mathrm{~s}$ after the actual release to allow autocalibration of the finger pressure device $[11,12]$, (4) the echo-tracking and finger BP devices were removed and the left forearm was fitted with the venous occlusion plethysmography, the BP measuring device being now positioned on the middle finger of the right arm, (5) following a 20-min interval forearm blood flow, BP and heart rate were measured for 15 min, (6) a suprasystolic pressure was applied for 12 min to the left arm to occlude the brachial artery and in the last 2 min of the occlusion period the subjects were asked to rhythmically clench and relax the left hand, (7) forearm blood flow, BP and heart rate were again measured during the $5 \mathrm{~min}$ which followed the release of the occlusion, (8) the 13 familial hypercholesterolemic subjects were maintained on a low lipid diet and given simvastatin at a dose of $40 \mathrm{mg}$ once daily. Only 7 (4 males and 3 females, age $46.1 \pm 3.0$ years) of these 13 patients agreed to participate to a 2 nd evaluation after a 6 month treatment, (9) in 
the same 7 subjects a third study was carried out after 24 months of dietary and simvastatin treatment. Seven subjects ( 4 males and 3 females, age $45.6 \pm 2.7$ years) from the control group were also studied again after a 6-month period.

RA diameter, compliance and distensibility values were always averaged over consecutive periods of $4 \mathrm{~s}$. Baseline diameter-pressure, compliance-pressure and distensibility-pressure curves were obtained by averaging 5 periods of 4 $\mathrm{s}$ taken at 3-min intervals during the 15 -min periods indicated at (2) (see above). Post-ischemic diameter-pressure, compliance-pressure and distensibility-pressure curves were obtained by taking the 4-s period showing the maximal increase in diameter following the release of brachial artery occlusion. Baseline blood flow was obtained by averaging 5 consecutive values over the 2.5 min preceeding brachial artery occlusion while post-ischemic forearm blood flow was obtained by taking the single maximal blood flow within the minute following the release of brachial artery occlusion. BP, heart rate and FVR were calculated at corresponding times. Total serum cholesterol, LDL cholesterol, high-density lipoprotein (HDL) cholesterol and triglycerides were measured from a blood sample taken from an antecubital vein in the morning preceeding the study. In familial hypercholesterolemic subjects lipid profile was also determined before wash-out from dietary and drug treatment.

Data from individual subjects were averaged and expressed as mean \pm S.E.M. for each group. The statistical significance of the differences in the means was assessed by the two-way analysis of variance. The two-tailed $t$-test for paired or unpaired observations was used to locate differences between the various conditions and the two groups, respectively. RA diameter was compared at the value corresponding to diastolic BP. To statistically compare RAC and distensibility, calculations were made of the integral of the area under the curve relating compliance and distensibility to the arterial BP normalized for the BP range from diastole to systole to take into account between-subject differences in pulse pressure value [14]. This was referred to as 'compliance index' and 'distensibility index'. A $P$ value $<0.05$ was taken as the level of statistical significance. Throughout the study the symbol \pm refers to the standard error of the mean.

\section{Results}

\subsection{Baseline BP, heart rate and lipid profile}

Table 1 shows that normocholesterolemic controls and familial hypercholesterolemic subjects had similar BP and heart rate values. Compared to control subjects total serum and LDL cholesterol were elevated in familial hypercholesterolemic subjects before wash-out from drug treatment, a further small although significant increase being observed after wash-out from drug treatment. HDL cholesterol and triglycerides were similar in the control and familial hypercholesterolemic groups, both before and after wash-out from drug therapy.

Table 1

Hemodynamic and lipid profile values in normocholesterolemic (C) and familial hypercholesterolemic subjects (FHC)

\begin{tabular}{|c|c|c|c|}
\hline \multirow[t]{2}{*}{ Variable } & \multirow[t]{2}{*}{$\mathrm{C}(n=10)$} & \multicolumn{2}{|c|}{ FHC $(n=13)$} \\
\hline & & $\begin{array}{l}\text { Before } \\
\text { wash-out }\end{array}$ & $\begin{array}{l}\text { After } \\
\text { wash-out }\end{array}$ \\
\hline SBP (mmHg) & $115.2 \pm 7.3$ & $\#$ & $106.5 \pm 4.8$ \\
\hline $\mathrm{DBP}(\mathrm{mmHg})$ & $68.9 \pm 4.9$ & $\#$ & $60.0 \pm 3.2$ \\
\hline $\mathrm{HR}(\mathrm{b} / \mathrm{min})$ & $66.3 \pm 4.9$ & $\#$ & $76.3 \pm 3.0$ \\
\hline $\begin{array}{l}\text { Serum } \\
\text { cholesterol } \\
(\mathrm{mg} / \mathrm{dl})\end{array}$ & $212.1 \pm 6.4$ & $\begin{array}{l}388.2 \\
15.6^{*}\end{array} \pm$ & $\begin{array}{l}401.6 \pm \\
16.9^{*}, * *\end{array}$ \\
\hline $\begin{array}{l}\text { LDL } \\
\text { cholesterol } \\
(\mathrm{mg} / \mathrm{dl})\end{array}$ & $135.1 \pm 4.8$ & $\begin{array}{l}309.5 \\
16.2^{*}\end{array}$ & $\begin{array}{l}331.7 \pm \\
17.1^{*}, * *\end{array}$ \\
\hline $\begin{array}{l}\text { HDL } \\
\text { cholesterol } \\
(\mathrm{mg} / \mathrm{dl})\end{array}$ & $55.2 \pm 4.2$ & $\begin{array}{l}53.8 \pm \\
5.2\end{array}$ & $51.8 \pm 5.9$ \\
\hline $\begin{array}{l}\text { Triglycerides } \\
(\mathrm{mg} / \mathrm{dl})\end{array}$ & $108.5 \pm 18.5$ & $\begin{array}{l}123.4 \pm \\
14.2\end{array}$ & $109.5 \pm 8.9$ \\
\hline
\end{tabular}

SBP, systolic blood pressure; DBP, diastolic blood pressure; $H R$, heart rate.

Data are expressed as means \pm S.E.M. ${ }^{*} P<0.01$ vs. N; ${ }^{* *} P$ $<0.01$ vs. FHC before wash-out. 

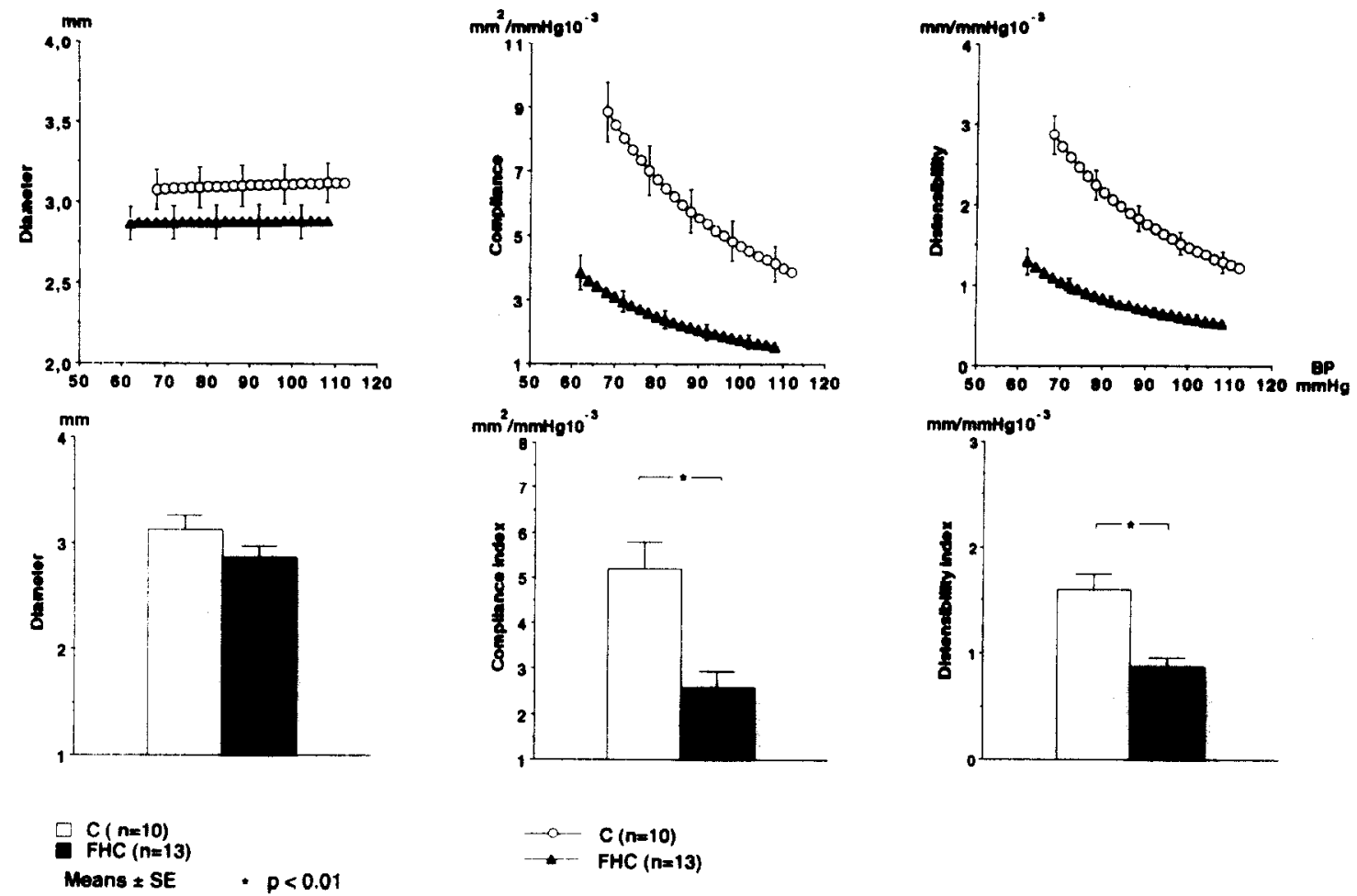

Fig. 1. Baseline radial artery diameter, compliance and distensibility in normocholesterolemic subjects $\mathrm{C}(n=10)(\mathrm{O} / \square)$ and in subjects with FHC $(n=13)(\boldsymbol{\Delta} / \mathbf{c})$. In the upper panels values are shown over the systo-diastolic blood pressure range. In the lower panels diameter is shown at diastolic blood pressure only while compliance and distensibility are shown as compliance index and distensibility index (see Methods). Data are shown as means \pm S.E.M.; ${ }^{*} P<0.05$.

\section{2. $R A C$ and distensibility}

As shown in Fig. 1, upper panels, in both control subjects and familial hypercholesterolemic subjects, an increase in BP from diastolic to systolic values was associated with a small progressive increase in RA diameter and a marked and progressive reduction in RAC and distensibility. RA diameter was slightly less in familial hypercholesterolemic subjects than in controls, although the difference at diastolic BP was not statistically significant (Fig. 1, lower left panel). In contrast, in familial hypercholesterolemics RAC and distensibility were strikingly reduced as compared to controls, the reduction being evident throughout the systo-diastolic pressure range. The compliance and distensibility indexes were less than half in the familial hypercholesterolemic than in the control group (Fig. 1, right panels).

Fig. 2 shows that in control subjects $\mathrm{R} \Lambda$ diameter, compliance and distensibility increased markedly after release from forearm ischemia throughout the systo-diastolic pressure range. In contrast, RA diameter, compliance and distensibility showed a smaller and non-significant increase in familial hypercholesterolemic subjects. In both groups BP was not affected by forearm ischemia.

\subsection{Maximal forearm vasodilatation}

Fig. 3 shows that in control and familial hypercholesterolemic subjects baseline forearm blood flow and vascular resistance were similar. In con- 

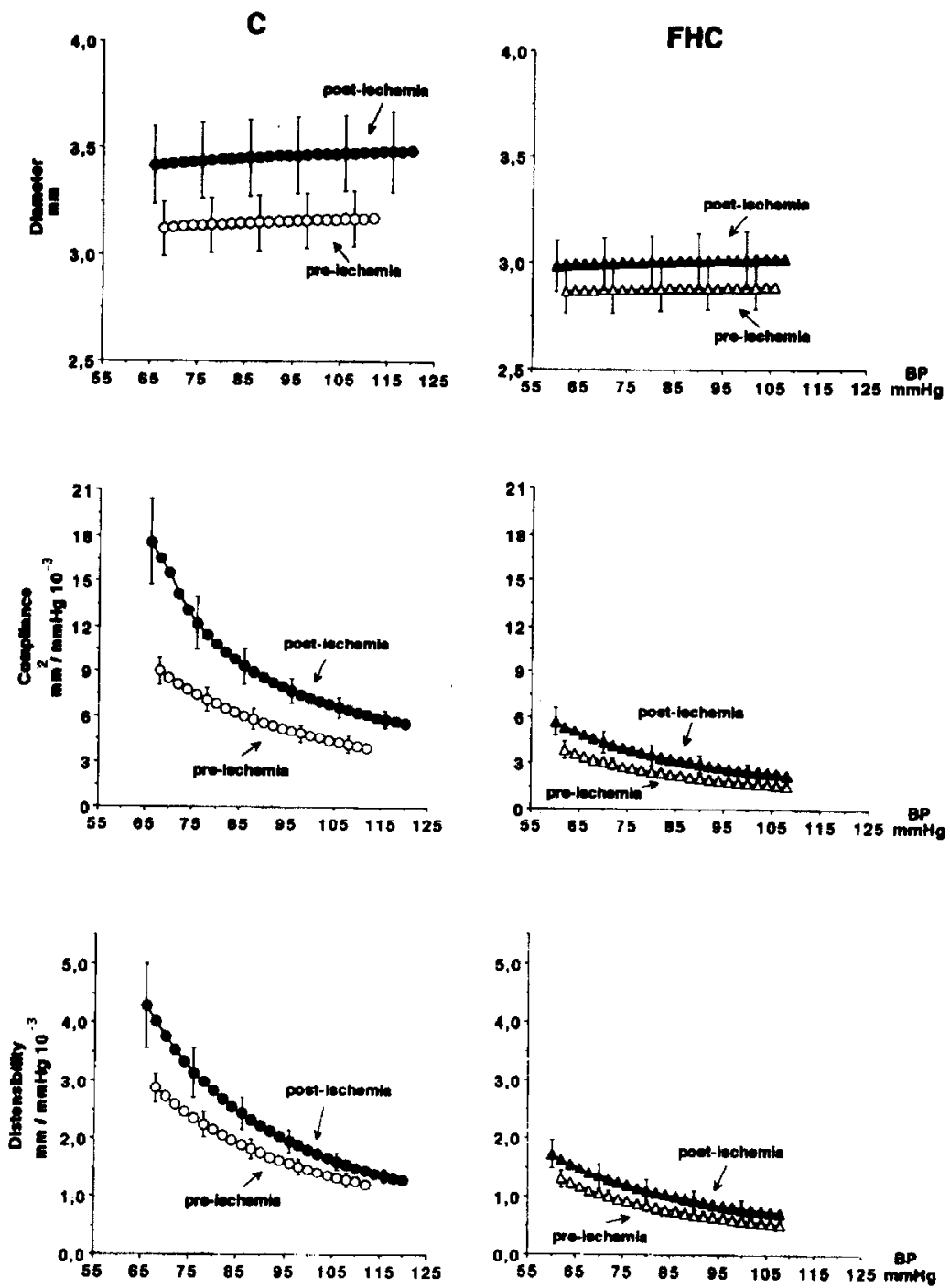

Fig. 2. Radial artery diameter, compliance and distensibility-pressure curves before (pre-ischemia) and after release from ischemia in the two groups of subjects of Fig. 1. Data are shown as means \pm S.E.M. (post-ischemia). Other explanations as in Fig. 1.

trol subjects release from prolonged forearm ischemia was associated with a marked increase in forearm blood flow and a marked reduction in FVR, whereas in familial hypercholesterolemic subjects both the increase in blood flow and the reduction in vascular resistance were much less pronounced. As a result, minimal FVR was much greater in the familial hypercholesterolemic than in the control group.

\subsection{Lipid lowering treatment}

As shown in Table 2 in control subjects BP, heart rate and lipid profile were similar in the two studies performed over a 6-month interval. In familial hypercholesterolemic subjects $\mathrm{BP}$ and heart rate were similar before and after 6- and 24-month lipid lowering drug treatment. Total serum cholesterol and LDL cholesterol were 

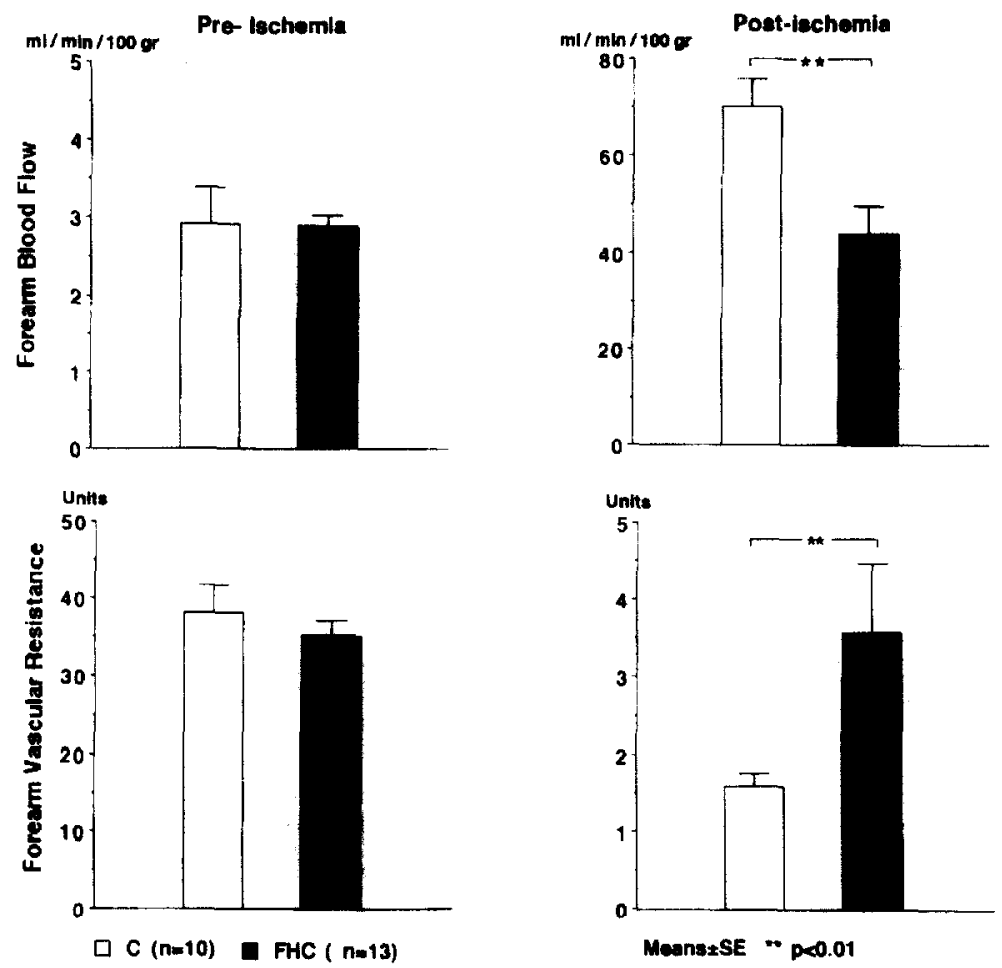

Fig. 3. Forearm blood flow and vascular resistance in the two groups of subjects of Fig. 1. Data (means \pm S.E.M.) are shown at baseline condition and during the vasodilatation occurring after the release from ischemia.

clearly reduced by lipid lowering treatment after 6 months and the reduction was even more pronounced after 24 months. Both after 6 and after 24 month lipid lowering treatment caused a small and non-significant increase of FIDL cholesterol leaving triglycerides unaffected.

The effects of lipid lowering treatment on maximal forearm blood flow and minimal FVR are shown in Fig. 4. In control subjects maximal forearm blood and minimal FVR were not significantly different in the two studies performed over a 6-month interval. In familial hypercholesterolemic subjects maximal forearm blood flow was significantly increased and minimal FVR was significantly reduced after 6 months of lipid lowering treatment and the reduction became even more pronounced when treatment was prolonged to 24 months. As shown in Fig. 4 (lower panels) and in Fig. 5 (upper panels) in control subjects RAC and distensibility pressure curves and indexes were similar in the two studies performed over the 6-month interval. Pre-ischemic RAC and distensibility were not significantly altered by 6 months of lipid lowering treatment also in familial hypercholesterolemic subjects. After 24 months of lipid lowering treatment, however, pre-ischemic compliance and distensibility values were increased, the difference in compliance and distensibility indexes being statistically significant. Post-ischemic compliance and distensibility values were slightly increased by the 6and 24-month lipid lowering treatment but the changes were less evident and not statistically significant (Fig. 4, lower panels and Fig. 5, lower panels). There was never a significant correlation between blood flow, compliance and distensibility values either before or after ischemia ( $r$ never $>0.39$ ). 
Table 2

Hemodynamics and lipid profile in normocholesterolemic controls (C) studied before and after 6 months and in subjects with FHC studied before and after 6 and 24 months of lipid lowering treatment

\begin{tabular}{|c|c|c|c|c|c|}
\hline \multirow[t]{2}{*}{ Variable } & \multicolumn{2}{|l|}{$\mathrm{C}(n=7)$} & \multicolumn{3}{|l|}{$\mathrm{FHC}(n=7)$} \\
\hline & First study & After 6 months & First study & Treatment 6 months & Treatment 24 months \\
\hline SBP (mmHg) & $111.0 \pm 9.9$ & $118.0 \pm 5.3$ & $100.8 \pm 6.5$ & $108.3 \pm 5.8$ & $111.7 \pm 11.1$ \\
\hline $\mathrm{DBP}(\mathrm{mmHg})$ & $65.8 \pm 5.1$ & $71.0 \pm 2.7$ & $55.8 \pm 3.2$ & $61.8 \pm 3.8$ & $61.6 \pm 8.5$ \\
\hline HR $(b / \min )$ & $64.0 \pm 5.9$ & $65.0 \pm 4.1$ & $72.1 \pm 3.1$ & $68.3 \pm 3.0$ & $72.8 \pm 2.8$ \\
\hline $\begin{array}{l}\text { Serum cholesterol } \\
(\mathrm{mg} / \mathrm{dl})\end{array}$ & $217.1 \pm 5.9$ & $220.0 \pm 6.7$ & $418.4 \pm 27.5^{*}$ & $341.0 \pm 33.3^{* * *}$ & $279.1 \pm 14.5^{*},{ }^{* *},{ }^{* * *}$ \\
\hline $\begin{array}{l}\text { LDL cholesterol } \\
(\mathrm{mg} / \mathrm{dl})\end{array}$ & $139.8 \pm 5.1$ & $129.0 \pm 9.2$ & $357.8 \pm 25.2^{*}$ & $271.0 \pm 30.1^{* * *}$ & $199.7 \pm 20.1^{*}, * *, * * *$ \\
\hline $\begin{array}{l}\text { HDL cholesterol } \\
(\mathrm{mg} / \mathrm{dl})\end{array}$ & $57.0 \pm 5.7$ & $58.0 \pm 5.7$ & $45.4 \pm 3.7$ & $46.7 \pm 2.5$ & $55.7 \pm 5.0$ \\
\hline Triglycerides (mg/dl) & $98.5 \pm 11.7$ & $93.7 \pm 12.1$ & $111.3 \pm 7.8$ & $125.7 \pm 17.3$ & $96.2 \pm 20.0$ \\
\hline
\end{tabular}

Data are expressed as means \pm S.E.M. Symbols and abbreviations as in Table $1 .{ }^{*} P<0.01$ vs. N; ${ }^{* *} P<0.01$ vs. first study, ***vs. 6 months treatment (FHC).

\section{Discussion}

Our results show that the marked elevation in serum cholesterol which characterizes FHC is accompanied by a marked reduction in the ability of forearm arterioles to maximally dilate following prolonged ischemia. They further show that this reduction is associated with a marked reduction in RAC and distensibility which become also unable to increase after prolonged ischemia, at variance from the pronounced increase seen in normocholesterolemic individuals. This allows to conclude that FHC is characterized by a striking impairment of resistance vessel dilatation. It is also characterized, however, by a striking impairment of the main function of arteries of greater caliber, i.e. by a reduction and a loss of modulation of arterial compliance and distensibility.

We can speculate that this alteration is caused by a reduction of arterial blood flow which reduces endothelial shear stress and thus secretion of endothelial factors increasing vessel wall compliance and distensibility via smooth muscle relaxation [19]. However, in familial hypercholesterolemic subjects baseline RAC and distensibility were strikingly reduced whereas baseline forearm blood flow was superimposable to that of control subjects. Furthermore, during the follow-up period the change in post-ischemic or maximal forearm blood flow did not proceed in parallel with the change in post-ischemic arterial compliance and distensibility. Neither in baseline condition nor after ischemia did the two sets of values show a significant correlation. This suggests that the reduction of RAC and distensibility of familial hypercholesterolemic subjects is at least in part due to abnormalities of the wall of this conduit vessel which is independent of the vessel diameter (reduction in distensibility) and thus intrinsic in nature. Three possibilities can be suggested. Firstly, in FHC there is an impaired secretion and/or function of endothelial relaxing factors independent of the flow regimen [19]. Secondly, the vessel wall is structurally altered by an increased deposition of cholesterol (a 'preatherosclerotic change') leading to an increased wall stiffness $[20,21]$. Thirdly, in this condition an increased concentration of oxidized lipoproteins [22] has a toxic vascular effect [23-26] that impairs arterial function.

Creager et al. [1], Chowienczyk et al. [2] and Casino et al. [3] have shown that in normotensive subjects with an average serum cholesterol of 275 $\mathrm{mg} / \mathrm{dl}, 300 \mathrm{mg} / \mathrm{dl}$ and $292 \mathrm{mg} / \mathrm{dl}$, respectively, the forearm dilatation induced by intra-arterial infusion of acetyl- or meta-choline was less than that 

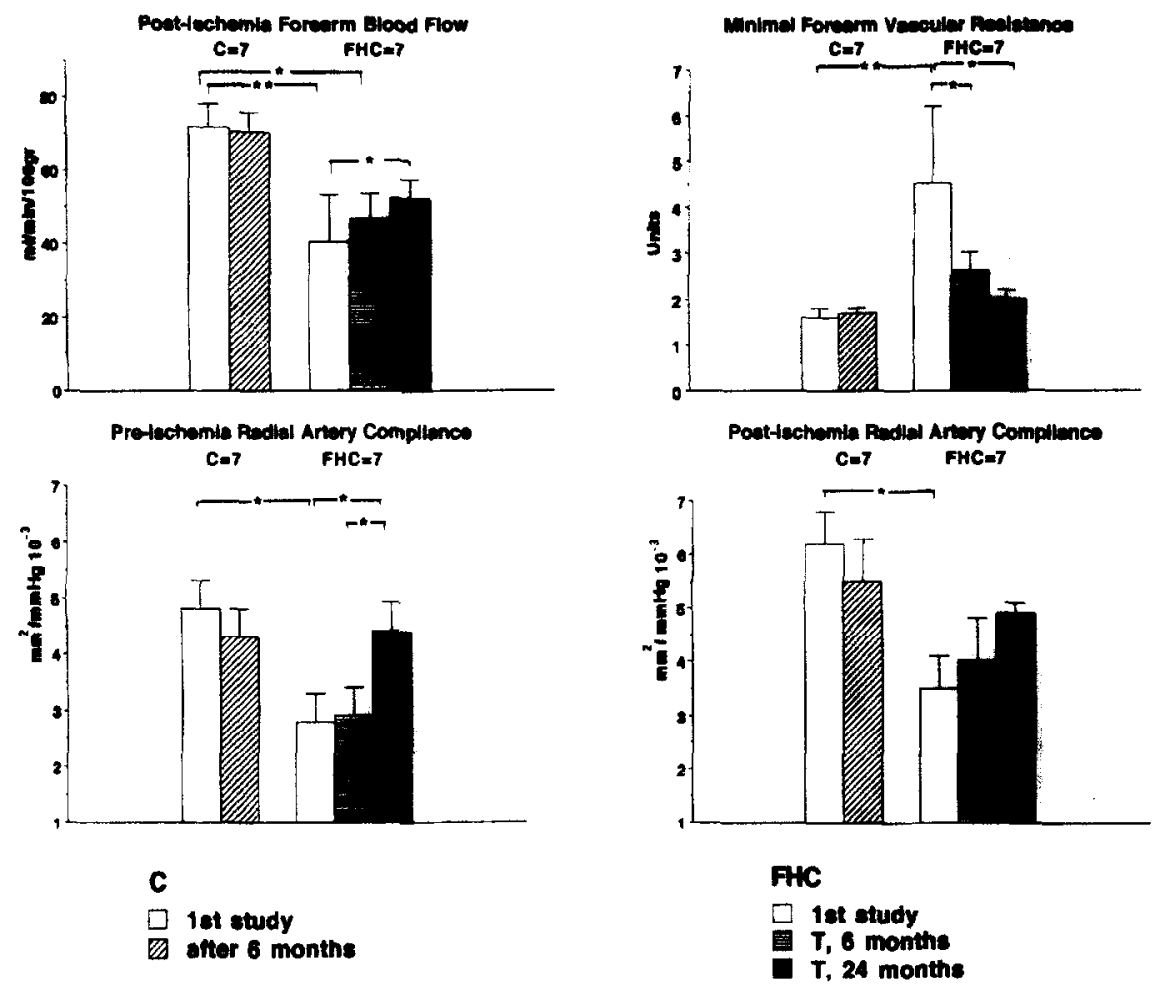

Fig. 4. Forearm blood flow, minimal vascular resistance and radial artery compliance index at baseline and after ischemia in FHC studied before (1st study) and after 6 and 24 months of lipid lowering treatment (T, 6 months; T, 24 months). Data from control (C) subjects studied twice at a 6-month interval are also shown. Mean \pm S.E.M. Symbols as in preceeding figures.

seen in normotensive normocholesterolemic controls, whereas the forearm vasodilatation induced by intra-arterial infusion of nitroprusside was similar in the two groups. This led to the conclusion that in hypercholesterolemia of a moderate degree the arteriolar modulation exerted by endothelial relaxing factors, whose secretion is promoted by acetyl- and meta-choline, is impaired, while the arteriolar modulation independent from these factors is normal [1-3]. Our study provides additional evidence on this issue because in our familial hypercholesterolemic subjects, in whom serum cholesterol was $401 \mathrm{mg} / \mathrm{dl}$, prolonged ischemia caused an arteriolar dilatation that was strikingly less than the one seen in control normocholesterolemic subjects. Because post-ischemic arteriolar dilatation is regarded as a 'non-endothelial' phenomenon (like the dilatation induced by nitroprussiate) [27], we suggest that while a moderate increase in serum cholesterol adversely affects the endothelial modulation of arteriolar tone only, a more marked increase in serum cholesterol impairs the overall ability of arterioles to dilate in a non-selective fashion. We also suggest that this is the case for the main function of larger arteries, i.e. compliance, whose marked post-ischemic increase was strikingly less in familial hypercholesterolemic subjects. An alternative possibility is that a non-selective and more marked loss of arteriolar dilatation and of arterial compliance increase is due to specific features of FHC affecting vascular wall properties, that are unrelated to serum cholesterol levels. However, this possibility is not supported by the evidence of a reversal of the arterial and arteriolar alterations induced by lipid lowering treatment.

In familial hypercholesterolemic subjects a 6 month treatment with simvastatin, that caused a 15.9 and $21.0 \%$ reduction in total and $\mathrm{LDL}$ cholesterol respectively, was accompanied by an 

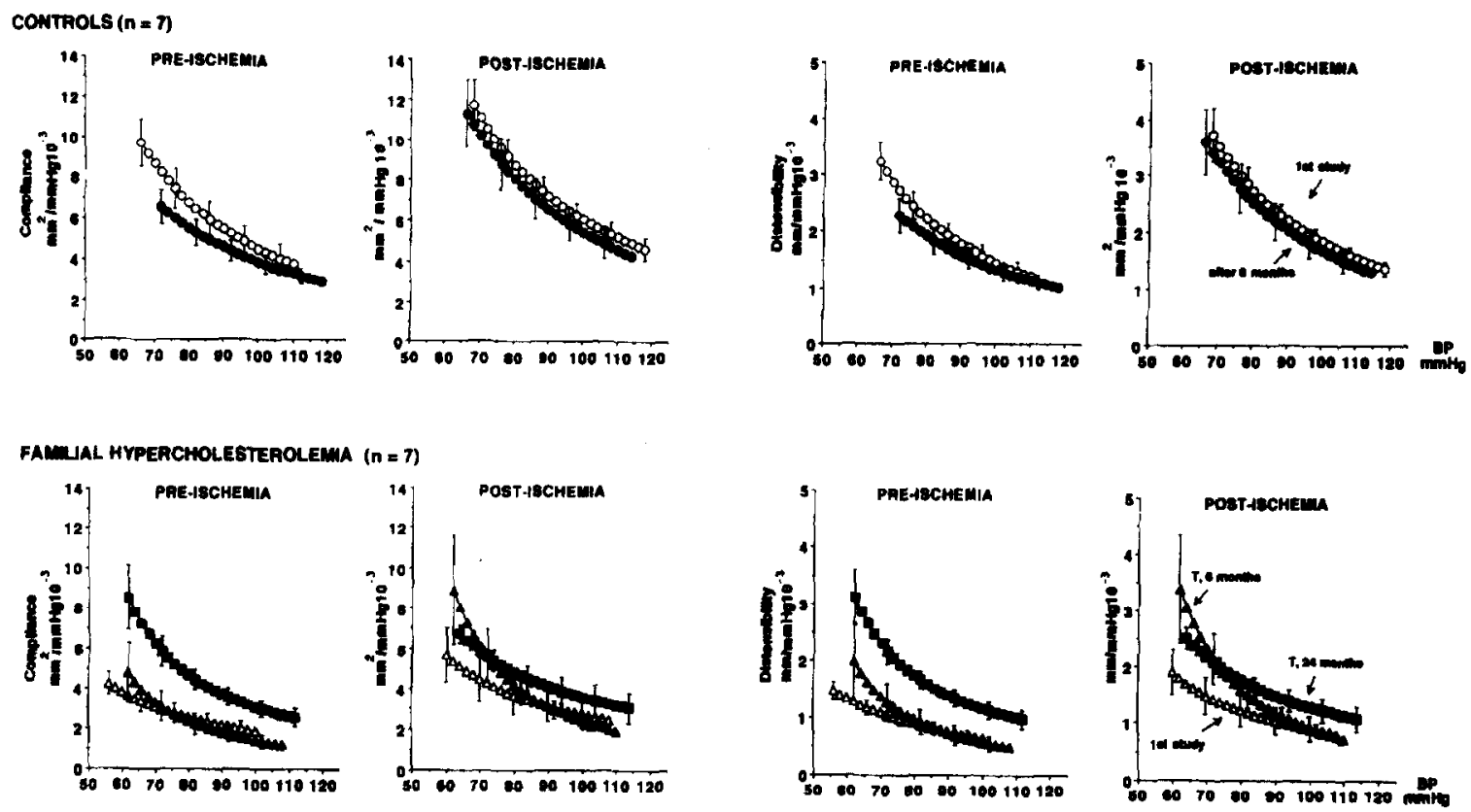

Fig. 5. Radial artery compliance and distensibility-pressure curves before and after ischemia in the subjects of Fig. 4. Symbols as in Fig. 4.

improvement in post-ischemic or maximal dilatation of forearm arterioles whereas baseline RAC increased in only a slight and non-significant fashion. However, when lipid lowering treatment was prolonged to 24 months and total and LDL cholesterol reduction was greater (20.7 and 40.9\% respectively), the improvement in maximal dilatation of forearm arterioles was slightly more pronounced, but a significant and clearcut increase in baseline RAC was observed as well. This allows to conclude that the marked alteration in arteriolar and arterial function seen in FHC is reversible by lipid lowering treatment. This goal, however, can be achieved if the reduction in total and LDL cholesterol is marked and long-lasting. This appears to be crucial particularly for the reversibility of the abnormality in RAC.

Our data are in line with the beneficial results on progression of atherosclerosis observed by normalizing lipid profile by means of a variety of lipid lowering interventions in subjects with FHC with a marked alteration in lipid profile [28-32]. They add to these results, however, the finding that the benefit extends to the basic functions of the arterial vessels, i.e. to the ability of the arteriole to dilate and of the larger artery to distend in response to physiological stimuli. Since lipid lowering treatment reduced minimal FVR already after 6 months, whereas the improvement in RAC was observed only after 24 months, in subjects with $\mathrm{FHC}$, reversal of the arteriolar impairment may be faster than reversal of the larger artery one. Twenty-four months of lipid lowering treatment did not significantly improve post-ischemic RAC. Thus, in FHC it may be more difficult or it may take longer to restore post-ischemic than baseline compliance values, suggesting that a reduced RAC reserve may thus remain a sign of an impaired compliance even during a long-term effective lipid lowering treatment. Our study does not answer the question whether in FHC large arteries with a predominant elastic structure share the striking reduction in compliance shown by the more muscular arteries such as the radial one. Studying large arteries compliance in FHC may pose special difficulties, however, because dynamic evaluation of compliance over the systo-diastolic pressure range is not yet possible in the aorta and 
other large arteries. Furthermore, at variance from the RA [33], large elastic arteries frequently undergo early atherosclerotic changes $[34,35]$, i.e. they frequently undergo non-specific structural damage which may superimpose on and thus prevent identification of more specific effects of FHC on arterial compliance [36,37].

Our results have clinical implications. An impaired arteriolar dilatation implies a potential danger for organs, such as the heart, which meet an increased metabolic requirement with an increase in blood flow, and indeed a reduced coronary reserve has been shown in normotensive subjects with a marked elevation in serum cholesterol [38-40]. Furthermore, an increased arterial stiffness may on the one hand reduce coronary blood flow during diastole and on the other hand increase aortic impedance, thereby offsetting the ratio between oxygen supply and demand of the heart [41]. An increased arterial stiffness may also increase the traumatic effect of BP on the arterial wall and thus favour the development of atherosclerosis. In animals this is exemplified by the diffuse atheromatic lesions displayed by carotid arteries in which stiffness is markedly increased by encasting vessels within rigid chambers [8]. Finally, even in subjects characterized by a marked alteration in lipid profile such as the familial hypercholesterolemic ones, treatment by lipid lowering drugs may reverse these arterial and arteriolar effects, thereby preventing the adverse consequences. To achieve this purpose, however, treatment must be effective over a long period.

\section{References}

[1] Creager M, Cooke JP, Mendelson ME, Gallagher SJ, Coleman SM, Loscalzo J, Dzau VJ. Impaired vasodilatation of forearm resistance vessels in hypercholesterolemic humans. J Clin Invest 1990;86:228-234.

[2] Chowienczyk PJ, Watts GF, Cockcroft JR, Ritte JM. Impaired endothelium-dependent vasodilatation of forearm resistance vessels in hypercholesterolemia. Lancet 1992;340:1430-1432.

[3] Casino PR, Kilcoyne CM, Quyyumi AA, Hoeg JM, Panza JA. The role of nitric oxide in endothelium-dependent vasodilation of hypercholesterolemic patients. Circulation 1993;88:2541-2547.
[4] Zelis R, Mason DT, Braunwald E, Levy RI. Effects of hyperlypoproteinemias and their treatment on the peripheral circulation. J Clin Invest 1970;49:1007-1015.

[5] Drexler H, Zeiher AM. Endothelial function in human coronary arteries in vivo: focus on hypercholestcrolemia. Hypertension 1991;18(II):II90-II99.

[6] Zeiher AM, Drexler H, Wollschlager H, Just H. Modulation of coronary vasomotor tone in humans: progressive endothelial dysfunction with different stages of coronary atherosclerosis. Circulation 1991;83:391-401.

[7] Ross R. The pathogenesis of atherosclerosis. An update. New Engl J Med 1986;314:488-500.

[8] Booth RFG, Martin JF, Honey AC, Hassal DG, Beesley JE, Moncada S. Rapid development of atherosclerotic lesions in the rabbit carotid artery induced by perivascular manipulation. Atherosclerosis 1989;76:257-268.

[9] Brown MS, Goldstein JL. Familial hypercholesterolemia: genetic, biochemical and pathophysiological considerations. Adv Intern Med 1979;20:273-276.

[10] Tardy Y, Meister JJ, Perret F, Brunner HR, Arditi M. Non invasive estimate of the mechanical properties of peripheral arteries from ultrasonic and photoplethysmographic measurements. Clin Phys Physiol Meas 1991:3:360-367.

[11] Parati G, Casadei R, Groppelli A, Di Rienzo M, Mancia G. Comparison of finger intra-arterial blood pressure monitoring at rest and during laboratory testing. Hypertension 1989;13:647-655.

[12] Imholz BP, Van Montfrans GA, Settels JJ, Van der Hoeven GM, Karemaker JM, Wieling W. Continuous non-invasive blood pressure monitoring: reliability of $\mathrm{Fi}$ napres device during the Valsalva maneuvre. Cardiovasc Res 1988;22(6):390-397.

[13] Langewouters GJ, Zwart A, Busse R, Wesseling KH. Pressure diameter relationship of segments of human finger arteries. Clin Phys Physiol Meas 1986;7:43-45.

[14] Giannattasio C, Mangoni AA, Stella ML, Carugo S, Grassi G, Mancia G. Acute effects of smoking on radial artery compliance in humans. J Hypertens 1994;12:691696.

[15] Giannattasio C, Cattaneo BM, Mangoni AA, Carugo S, Failla M, Trazzi S, Sega R, Grassi G, Mancia G. Cardiac and vascular structural changes in normotensive subjects with parental hypertension. J Hypertens 1995;13(2):259264.

[16] Giannattasio C, Failla M, Stella ML, Mangoni AA. Carugo S, Pozzi M. Grassi G, Mancia G. Alterations in radial artery compliance in patients with congestive heart failure. Am J Cardiol 1995;76:381-385.

[17] Hayoz D, Drexler H, Munsen T, Harnig B, Zeiler AH, Just H, Brunner HR, Zelis R. Flow-mediated arterial dilation is abnormal in congestive heart failure. Circulation 1993;87(7):VII-92-VII-96.

[18] Folkow B, Grimby G, Thulesius O. Adaptive structural changes of the vascular walls in hypertension and their relations to the control of peripheral resistance. Acta Physiol Scand 1958:44:225 -272. 
[19] Furchgott RF, Zawadski JV. The obligatory role of endothelial cells in the relaxation of arterial smooth muscle by acetylcholine. Nature 1980;299:373-376.

[20] Scott-Burden T, Resink TJ, Hann AWA, Baur U, Box RJ, Bunler FR. Induction of growth-related metabolism in human vascular smooth muscle cells by low-density lipoprotein. J Biol Chem 1989;264:12582-12589.

[21] Ross R, Glomset J. Atherosclerosis and the arterial muscle cell: proliferation of smooth muscle is a key event in the genesis of the lesions of atherosclerosis. Science 1973;180:1332-1339.

[22] Genest JJ Jr, Martin-Munley SS, McNamara JR, Ordovas JM, Jenner J, Myers RH, Silberman SR, Wilson P, Salam DN, Schaefer EJ. Familial lipoprotein disorders in patients with premature coronary artery disease. Circulation 1992;85(6):2025-2033.

[23] Andrews HE, Bruckdorfer KR, Dunn RC, Jacops M. Low density lipoproteins inhibit endothelial dependent relaxation in rabbit aorta. Nature 1987;3278:237-239.

[24] Kugiyama K, Kerns SA, Morrisett JD, Roberts R, Henry PD. Impairment of endothelium-dependent relaxation by lysolecithin in modified low-density lipoproteins. Nature 1990;344:160-162.

[25] Matsuda $Y$, Hirata $K$, Inoue $N$, Suematsu $M$, Kawashima S, Akita H, Yokoyama M. High density lipoprotein reverses inhibitory effect of oxidized low density lipoprotein on endothelium-dependent arterial relaxation. Circ Res 1993;72:1103-1109.

[26] McLenachan JM, Williams JK, Fish RD, Ganz P, Selwyn AP. Loss of flow-mediated endothelium-dependent dilatation occurs early in the development of atherosclerosis. Circulation 1991;84:1273-1278.

[27] Skinner SN. Skeletal Muscle Blood Flow. Metabolic determinants. In Zelis R, (ed.). The Peripheral Circulation. New York: Grune Stratton 1975:57-78.

[28] Brown G, Albers JJ, Fischer LD. Regression of coronary artery disease as a result of intensive lipid-lowering therapy in men with high levels of apolipoprotein B. New Engl J Med 1990;323:1289-1298.

[29] Bissett JK, Wyeth RP, Matts JP, Johnson JW. Plasma lipid concentrations and subsequent coronary occlusion after a first myocardial infarction. The POSCH Group. Am J Med Sci 1993;305 (3):139-144.

[30] Koga N, Iwata Y. Pathological and angiographic regression of coronary atherosclerosis by LDL-apheresis in a patient with familial hypercholesterolemia. Atherosclerosis $1991 ; 90(1): 9-21$.
[31] Brown BG, Lin JT, Passamani ER, Levy RI, Dodge HT, Detre KM. Progression of coronary atherosclerosis in patients with probable familial hypercholesterolemia. Quantitative arteriographic assessment of patients in NHLBI type II study. Arteriosclerosis 1989;9(1):181-190.

[32] Egashira K, Hirooka Y, Kai H, Sugimachi M, Suzuchi M, Inou T, Takeshita A. Reduction in serum cholesterol with pravastatin improves endothelium-dependent coronary vasomotion in patients with hypercholesterolemia. Circulation 1994;89:2519-2524.

[33] McGill HC Jr. The Geographic Pathology of Atherosclerosis. Baltimore: Williams and Wilkins, 1968.

[34] Riley WA, Freedman DS, Higgs NA, Barncs RW, Zinkgraf SA, Berenson GS. Decreased arterial elasticity associated with cardiovascular disease risk factors in the young. Bogalousa HART study. Arteriosclerosis 1986;6:378-386.

[35] Lehmann ED, Watts GF, Fatemi-Langroudi B, Gousling RG. Aortic compliance in young patients with heterozygous familial hypercholesterolemia. Clin Sci 1982;83:717-721.

[36] Wendelhag I, Wilkund O, Wilkstrand J. Atherosclerotic changes in the femoral and carotid arteries in familial hypercholesterolemia. Ultrasonographic assessment of intima-media thickness and plaque occurrence. Atheroscler Thromb 1993;13:1404-1410.

[37] Gariepy J, Simon A, Massonreau M, Linhart A, Levenson J. Wall thickening of carotid and femoral arteries in male subjects with isolated hypercholesterolemia. Atherosclerosis 1995;113:141-151.

[38] Forstermann U, Mugge A, Alheid U, Haverich A, Frolich JC. Selective attenuation of endothelium-mediated vasodilatation in atherosclerotic human coronary arteries. Circ Res 1988;62:185-190.

[39] Ludmer PL, Selwyn AP, Shook TL, Wayne RR, Mudge GH, Alexander RW, Ganz P. Paradoxical vasoconstriction induced by acetylcholine in atherosclerotic coronary arteries. New Engl J Med 1986;315:1046-1051.

[40] Vita JA, Treasure CB, Nabel EG, McLenachan JM, Fish RD, Yeung AC, Vekstein VI, Selwyn AP, Ganz P. Coronary vasomotor response to acetylcholine relates to risk factors for coronary heart disease. Circulation 1990;81:491-497.

[41] O'Rourke MF. Arterial function in health and disease. Edinburgh: Churchill Livingstone, 1982. 